\title{
TO DEVELOP FORENSIC CRITERIA FOR THE DIFFERENTIAL DIAGNOSIS OF INTRACEREBRAL HEMORRHAGE OF TRAUMATIC AND NON-TRAUMATIC ORIGIN USING MUELLER-MATRIX MICROSCOPY OF LINEAR DICHROISM
}

\author{
Marta Garazdiuk $^{1}$, Viktor Bachynskyi ${ }^{2}$, Olena Nechytailo ${ }^{3}$, Oleksandr Garazdiuk ${ }^{4}$
}

\begin{abstract}
Differential diagnosis of intracerebral hemorrhage due to traumatic and non-traumatic origin is a challenging issue, especially in the absence of visible body injuries and other signs of violence. For a forensic expert-practitioner, the main thing is objectivity, accuracy, and speed of obtaining the result, which could fully satisfy the methods of laser polarimetry in the case of differential diagnosis of hemorrhages of traumatic and non-traumatic origin in the human brain matter.

The purpose: To develop forensic criteria for the differential diagnosis of intracerebral hemorrhage of traumatic and nontraumatic origin using Mueller-matrix microscopy of linear dichroism.
\end{abstract}

Materials and methods: The object of the study was brain matter samples, collected in 115 corpses of both sexes aged 22 to 86 years with accurately known causes of death. The causes of death included coronary artery disease, traumatic brain hemorrhage, ischemic brain infarction, and non-traumatic brain hemorrhages. Brain matter samples have been frozen rapidly, and histological sections have been made using a freezing microtome. The slices have been evaluated using the method of azimuthal-invariant Mueller-matrix microscopy, followed by mathematical and statistical processing of the results.

Results: significant topographic heterogeneity of Mueller-matrix invariants of brain matter samples linear dichroism maps among all research groups have been found. Individual and significant variations of dispersion, asymmetry, and kurtosis magnitude have confirmed the heterogeneity of Mueller-matrix invariants. The difference of images and histograms among groups is explained by the presence of necrotic changes in the case of ischemic brain necrosis and blood cells in the brain matter samples. For the set of central statistical moments of the 1st, 3rd, and 4th orders, which characterize the distributions of circular dichroism, the method of MM-microscopy in the differentiation of samples of non-hemorrhage and hemorrhage groups reaches a satisfactory level $-78 \%-84 \%$.

Conclusions: Brain matter samples Muller-matrix microscopy of linear dichroism is effective for solving diagnostic problems of forensic medicine related to evaluating the cause of death from intracerebral hemorrhage of various origins. Diagnostic efficiency of Mueller-matrix mapping of polarization manifestations of linear dichroism method for intergroup differentiation of samples of deaths from traumatic hemorrhage (Group 2) and ischemic cerebral infarction (Group 3) reaches a satisfactory level of $79-84 \%$.

UDC Classification: 616.1, DOI: https://doi.org/10.12955/pmp.v2.165

Keywords: traumatic intracranial hemorrhage, non-traumatic intracranial hemorrhage, cause of death, azimuthal-invariant Mueller-matrix microscopy

\section{Introduction}

Forensic pathology of trauma is a complex and extremely important area of forensic science and is dedicated to evaluating the causes, mechanisms, and outcomes of traumatic injuries (Bertozzi et al., 2020). Traumatic brain hemorrhage (TBH) is one of the most common injuries. Consequently, the identification and diverse morphological assessment of injuries to the skull bones and brain matter require a methodologically correct approach (Finnie, 2016). Differential diagnosis of intracerebral hemorrhage due to traumatic and non-traumatic origin is a challenging issue, especially in the absence of visible body injuries and other signs of violence (Hao \& Wang, 2017).

Physical-optical methods based on laser irradiation of biological tissues demonstrated their high sensitivity and efficacy in forensic practice (Trifonyuk et al., 2020). Bachinsky VT (Bachinsky et al., 2021) corroborated the role of Mueller-matrix analysis in the differential diagnosis of acute myocardial ischemia and chronic coronary artery disease. Sivokorovskaya A. (Sivokorovskaya et al., 2020) demonstrated the role of polarization-phase diagnostics of the volume of blood loss.

Our previous studies demonstrated the efficacy of thin-layer polarization tomography to differentiate the origin of hemorrhage into the brain (Garazdiuk et al., 2020). For a forensic expert-practitioner, the main thing is objectivity, accuracy, and speed of obtaining the result, which could fully satisfy the

\footnotetext{
${ }^{1}$ Bukovinian State Medical University, Department of Forensic Medicine and Medical Law, Chernivtsi, Ukraine, m.garazdiuk@gmail.com, ORCID 0000-0003-1536-4440

${ }^{2}$ Bukovinian State Medical University, Department of Forensic Medicine and Medical Law, Chernivtsi, Ukraine, sudmed@bsmu.edu.ua, ORCID 0000-0002-6955-7507

${ }^{3}$ Bukovinian State Medical University, Department of anesthesiology and reanimatology, Chernivtsi, Ukraine, olena.nechytailo@bsmu.edu.ua, ORCID 0000-0003-3932-4902

${ }^{4}$ Bukovinian State Medical University, Internal Medicine and Infectious Diseases Department, Chernivtsi, Ukraine, olexandr.harazdiuk@gmail.com, ORCID 0000-0002-0430-7558
} 
methods of laser polarimetry in the case of differential diagnosis of hemorrhages of traumatic and nontraumatic origin in the human brain (Hao \& Wang, 2017).

\section{The purpose of the work}

To develop forensic criteria for the differential diagnosis of intracerebral hemorrhage of traumatic and non-traumatic origin using Mueller matrix (MM) microscopy of linear dichroism (LD).

\section{Materials and methods}

The object of the study was human brain matter samples (BMS) collected in 115 corpses of both sexes aged 22 to 86 years with accurately known causes of death. The causes of death included coronary artery disease (no signs of intracranial hemorrhage), TBH (visible life-threatening changes to the skull bones and brain), ischemic brain infarction (according to criteria, described by (Bailey et al., 2012)) and nontraumatic brain hemorrhage (intravital diagnosis of hemorrhagic stroke). The samples have been divided into 4 research groups, depending on the cause of death: coronary artery disease (CAD) - Group 1 - 20 samples (17.4\%), TBH - Group 2 - 30 samples (26.1\%), ischemic brain infarction (IBI) - Group 3 - 35 samples $(30,4 \%)$, non-traumatic brain hemorrhages (NTBH) - Group 4 - 30 samples (26.1\%). According to the absence of significant morphological changes in brain matter among subjects with CAD, Group 1 was considered the control group. BMS has been frozen rapidly, and histological sections (thickness of each slice $-0,4 \mathrm{mcm}$ ) have been made using a freezing microtome. The slices have been evaluated using the method of azimuthal-invariant MM-microscopy, followed by mathematical and statistical processing of the results.

The description of the laser polarimeter and its modifications and methods for measuring the coordinate distributions of polarization parameters, are detailed in scientific papers (Ushenko, 2011). Statistical evaluation included the mean (statistical moment $(\mathrm{SM})$ of the first order $\left(\mathrm{SM}_{1}\right)$ ), dispersion (SM of the second-order $\left(\mathrm{SM}_{2}\right)$ ), asymmetry $\left(\mathrm{SM}_{3}\right)$, and sharpness of the peak (kurtosis) of coordinate distributions $\left(\mathrm{SM}_{4}\right)$ of polarization and fluorescence image maps calculation for each BMS image. Results have been presented as mean and its standard deviation.

The evaluation of the volumetric polycrystalline structure of BMS histological slices included the following experimental and algorithmic steps:

- application of an additional reference laser wave superimposed on the polarizationinhomogeneous microscopic image of the depolarizing BMS.

- digital holographic reproduction of layer-by-layer distribution sets of azimuthal-invariant Mueller-matrix parameters, which characterize the polarizing distributions of circular birefringence;

- elaboration of forensic criteria to evaluate the efficacy of the MM of LD method of BMS for objective differentiation among different causes of death.

\section{Results and discussion.}

The topographic maps of MM azimuthal-invariant mapping results (fragments (1), (3), (5), (7)) and MM invariants of LD probability distributions histograms (fragments (2), (4), (6), (8) ) are presented at the series of fragments (Figure 1).

The next results have been obtained: significant topographic heterogeneity of MM invariants of LD maps of BMS in all groups (Fig. 1, fragments (1), (3), (5), (7)). Individual and significant variations of the $\mathrm{SM}_{2}-\mathrm{SM}_{4}$ magnitude (Fig. 1, fragments (2), (4), (6), (8)) confirm heterogeneity of MM invariants.

The difference of images and histograms between Group 1 and Group 3 could be explained by the presence of necrotic changes in brain matter in patients with IS (Trifonyuk et al., 2020) whereas such morphological changes are absent in CAD subjects. In Group 2 and Group 4, difference is determined by the presence of blood cells in the BMS (Kaur \& Sharma, 2018). Table 1 demonstrates the results of MM invariants LD maps statistical analysis.

Comparison of statistical analysis revealed diagnostic sensitivity of the set of central statistical moments of 2nd - 4th orders between Group 1 and Groups 2-4. Along with this, the opportunity of intergroup (experimental groups 2 - 4) difference in MM invariants of LD has been established: between Group 3 and Group $4(\mathrm{p}<0.05)$ and between Group 2 and Group 3 ( $\mathrm{p}<0.05)$.

At the next step of the research, we analyzed the strength of the azimuthal-invariant MM polarimetry method to distinguish the cause of death between research groups. An increase in the value of sensitivity 
$(\mathrm{Se}, \%)$, specificity $(\mathrm{Sp}, \%)$, and balanced accuracy $(A c, \%)$ in $14 \%-19 \%$ compared to the data of previous MM-mapping researches of TMS (Garazdiuk et al., 2020).

Figure 1: Topographic maps (1), (3), (5), (7) and histograms (2), (4), (6), (8)) of MM of LD distribution of BMS.

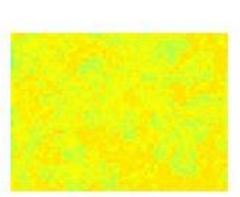

(1)

Group 1

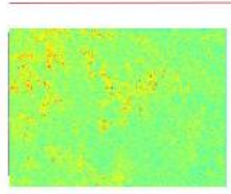

(5)

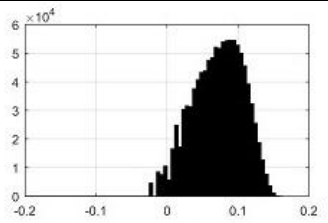

(2)

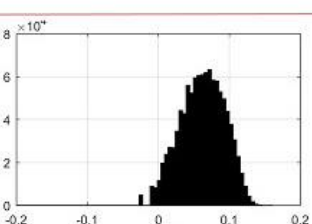

(6)

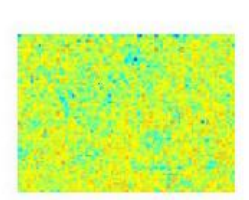

(3)

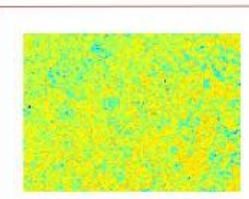

(7)

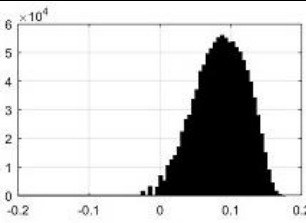

(4)

Group 2

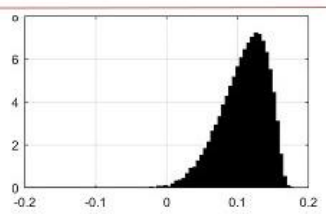

(8)

Source: Authors

Table 1: Central statistical moments of the 1st - 4th orders, which characterize the maps of BMS MM invariants of LD distribution

\begin{tabular}{|c|c|c|c|c|}
\hline Values & Group 1 & Group 2 & Group 3 & Group 4 \\
\hline Mean, SM $_{1}$ & $0.24 \pm 0.009$ & $0.22 \pm 0.011$ & $0.25 \pm 0.01$ & $0.21 \pm 0.008$ \\
\hline $\mathbf{p}_{1}$ & & $\mathrm{p}<0.05$ & $\mathrm{p}<0.05$ & $\mathrm{p}<0.05$ \\
\hline $\mathbf{p}_{2}$ & & \multicolumn{2}{|c|}{$\mathrm{p}>0.05$} & $p>0.05$ \\
\hline $\mathbf{p}_{3}$ & & $\mathrm{p}>0.05$ & \multicolumn{2}{|c|}{$p>0.05$} \\
\hline $\mathbf{p}_{4}$ & & \multicolumn{3}{|c|}{$\mathrm{p}>0.05$} \\
\hline Dispersion, $\mathbf{S M}_{2}$ & $0.21 \pm 0.011$ & $0.27 \pm 0.017$ & $0.32 \pm 0.016$ & $0.37 \pm 0.014$ \\
\hline p1 & & $\mathrm{p}<0.05$ & $\mathrm{p}<0.05$ & $\mathrm{p}<0.05$ \\
\hline $\mathbf{p}_{2}$ & & \multicolumn{2}{|c|}{$p>0.05$} & $p>0.05$ \\
\hline $\mathbf{p}_{3}$ & & $p>0.05$ & \multicolumn{2}{|c|}{$\mathrm{p}>0.05$} \\
\hline p4 & & \multicolumn{3}{|c|}{$p>0.05$} \\
\hline Asymmetry $\mathrm{SM}_{3}$ & $0.69 \pm 0.027$ & $0.87 \pm 0.034$ & $0.98 \pm 0.035$ & $1.11 \pm 0.044$ \\
\hline $\mathbf{p}_{1}$ & & $\mathrm{p}<0.05$ & $p<0.05$ & $\mathrm{p}<0.05$ \\
\hline $\mathbf{p}_{2}$ & & \multicolumn{2}{|c|}{$p>0.05$} & $\mathrm{p}<0.05$ \\
\hline $\mathbf{p}_{3}$ & & $\mathrm{p}>0.05$ & \multicolumn{2}{|c|}{$p>0.05$} \\
\hline $\mathbf{p}_{4}$ & & \multicolumn{3}{|c|}{$\mathrm{p}>0.05$} \\
\hline Kurtosis, SM4 & $0.89 \pm 0.041$ & $1.12 \pm 0.048$ & $1.22 \pm 0.051$ & $1.43 \pm 0.059$ \\
\hline $\mathbf{p}_{1}$ & & $\mathrm{p}<0.05$ & $\mathrm{p}<0.05$ & $\mathrm{p}<0.05$ \\
\hline $\mathbf{p}_{2}$ & & \multicolumn{2}{|c|}{$p>0.05$} & $p>0.05$ \\
\hline $\mathbf{p}_{3}$ & & $\mathrm{p}>0.05$ & \multicolumn{2}{|c|}{$p>0.05$} \\
\hline
\end{tabular}

Source: Author

For the set of central statistical moments of the 1st, 3rd, and 4th orders, which characterize the distributions of circular dichroism, the method of MM-microscopy in the differentiation of samples of non-hemorrhage and hemorrhage groups reaches a satisfactory level - 78\% - 84\%.

\section{Conclusion}

1. Brain matter samples Muller-matrix microscopy of linear dichroism is effective for solving diagnostic problems of forensic medicine related to evaluating the cause of death from intracerebral hemorrhage of various origins.

2. Diagnostic efficiency of Mueller-matrix mapping of polarization manifestations of linear dichroism method for intergroup differentiation of samples of deaths from traumatic intracerebral hemorrhage (Group 2) and ischemic cerebral infarction (Group 3) reaches a satisfactory level of 79 - 84\%. A similar 
satisfactory level (79-82\%) of differentiation is achieved for samples of histological sections of deaths from hemorrhagic (Group 4) and ischemic cerebral infarction (Group 3).

\section{References}

Bachinsky, V., Vanchulyak, Y., Ushenko, A. G., Ushenko, Y. A., Dubolazov, A. V., Bykov, A., Hogan, B., \& Meglinski, I. (2021). Diagnosis of Acute Coronary Insufficiency by the Method of Mueller Matrix Analysis of Myosin Myocardium Networks SpringerBriefs in Applied Sciences and Technology (pp. 53-87): Springer.

Bailey, E. L., Smith, C., Sudlow, C. L., \& Wardlaw, J. M. (2012). Pathology of lacunar ischemic stroke in humans-a systematic review. Brain Pathology, 22(5), 583-591. doi:10.1111/j.1750-3639.2012.00575.x

Bertozzi, G., Maglietta, F., Sessa, F., Scoto, E., Cipolloni, L., Di Mizio, G., Salerno, M., \& Pomara, C. (2020). Traumatic Brain Injury: A Forensic Approach: A Literature Review. Curr Neuropharmacol, 18(6), 538-550. doi:10.2174/1570159x17666191101123145

Finnie, J. W. (2016). Forensic Pathology of Traumatic Brain Injury. Vet Pathol, 53(5), 962-978. doi:10.1177/0300985815612155

Garazdiuk, M., Bachynskiy, V., Nechytailo, O., Garazdiuk, O., \& Malanchuk, S. (2020). Methods to Determine Complex Degrees of Mutual Anisotropy for the Differentiation of Hemorrhages in the Human Brain Substance Resulting From Traumatic and Non-traumatic Genesis. Proceedings of CBU in Medicine and Pharmacy, 1, 18-23. doi:10.12955/pmp.v1.92 Hao, S., \& Wang, B. (2017). Editorial: Review on Intracerebral Haemorrhage: Multidisciplinary Approaches to the Injury Mechanism Analysis and Therapeutic Strategies. Curr Pharm Des, 23(15), 2159-2160. doi: $10.2174 / 1381612823999170307150448$

Kaur, P., \& Sharma, S. (2018). Recent Advances in Pathophysiology of Traumatic Brain Injury. Curr Neuropharmacol, 16(8), 1224-1238. doi:10.2174/1570159x15666170613083606

Sivokorovskaya, N., Bachinsky, V., Vanchulyak, O., Ushenko, A., Dubolazov, A., Ushenko, Y., \& Tomka, Y. (2020). Polarization-phase diagnostics of volume of blood loss. Paper presented at the Applications of Digital Image Processing XLIII.

Trifonyuk, L., Sdobnov, A., Baranowski, W., Ushenko, V., Olar, O., Dubolazov, A., Pidkamin, L., Sidor, M., Vanchuliak, O., Motrich, A., Gorsky, M., \& Meglinski, I. (2020). Differential Mueller matrix imaging of partially depolarizing optically anisotropic biological tissues. Lasers Med Sci, 35(4), 877-891. doi:10.1007/s10103-019-02878-2

Ushenko, Y. A. (2011). Investigation of formation and interrelations of polarization singular structure and Mueller-matrix images of biological tissues and diagnostics of their cancer changes. J Biomed Opt, 16(6), 066006. doi:10.1117/1.3585689 